\begin{abstract}
Adriana Soares Torres Melo Maria Amélia Ribeiro Elias Aline Silva de Aguiar
\end{abstract}

'Pós-graduação em Saúde Coletiva, Faculdade de Medicina, Universidade Federal de Juiz de Fora, Brasil.

¿Unidade de Nutrição Clínica, Hospital Universistário, Universidade Federal de Juiz de Fora, Brasil.

${ }^{3}$ Departamento de Nutrição, Instituto de Ciências Biológicas, Universidade Federal de Juiz de Fora, Brasil.

\section{Aline de Aguiar}

Campus Universitário, rua José Lourenço Kelmer, s/n, São Pedro, Juiz de Fora, Minas Gerais

CEP: $36036-900$

จ aline.aguiar@ufjf.edu.br

\section{RESUMO}

Introdução: O controle da hiperfosfatemia é um desafio no tratamento de pacientes hemodialíticos, sendo um dos principais objetivos a serem alcançados. O acompanhamento nutricional e a prática de atividades educativas contribuem para o sucesso na adesão ao tratamento. Objetivo: Descrever, em indivíduos em hemodiálise, o conhecimento do tratamento da hiperfosfatemia, bem como avaliar o possível impacto das orientações nutricionais nos níveis séricos de fósforo após intervenção nutricional em participantes do programa de HD no Hospital Universitário na cidade de Juiz de Fora, MG. Material e Métodos: Estudo quase-experimental, do tipo antes e depois com 35 pacientes em tratamento hemodialítico, com 18 anos ou mais, de ambos os sexos, em diálise há, pelo menos, dois meses e que haviam passado por, pelo menos, uma consulta com a nutricionista do setor. Foram coletados do prontuário dados sociodemográficos e clínicos e aplicados questionário de frequência alimentar adaptado e recordatório $24 \mathrm{~h}$ para avaliação do consumo alimentar, além de questões fechadas sobre o conhecimento das causas da hiperfosfatemia, alimentos ricos em fósforo e uso de quelantes. A intervenção foi realizada mediante a utilização de cartilha educativa. Após a intervenção foram verificados os níveis séricos de fósforo, considerando hiperfosfatemia valores $>5,5 \mathrm{mg} / \mathrm{dL}$. Resultados: Na amostra, $57,1 \%(n=20)$ eram do sexo masculino, com média de idade de $61,8 \pm 14,3$ anos e tempo médio em diálise de $61,1 \pm$ 68,9 meses. A prevalência de hiperfosfatemia antes da intervenção era de $60 \%(n=21)$ e ao final da intervenção houve redução para $25,7 \%(n=9)$. Houve diferença na redução dos níveis séricos de fósforo quando comparadas as médias antes e depois da intervenção $[(5,9 \pm 1,3 \mathrm{mg} / \mathrm{dL} ; 4,9 \pm 1,7 \mathrm{mg} / \mathrm{dL} ; \mathrm{p}<0,001)]$. Conclusão: Os resultados mostraram que a intervenção educativa possivelmente contribuiu para melhorar a compreensão e adesão ao tratamento, tendo um papel complementar no manejo da hiperfosfatemia de pacientes dialíticos.

Palavras-chave: Educação Alimentar e Nutricional; Diálise Renal; Hiperfosfatemia; Insuficiência Renal Crônica.

\section{ABSTRACT}

Introduction: Hyperphosphatemia control is a challenge in the treatment of patients in hemodialysis, which is one of the main objectives to be reached. Nutritional accompaniment and practice of educational activities contribute to the success in the adhesion to the treatment. Objective: Describe, in individuals in hemodialysis, the knowledge of the treatment of hyperphosphatemia, as well as to evaluate the possible impact of nutritional orientations on phosphorus levels after nutritional intervention in participants of the program HD at the University Hospital in the city of Juiz de Fora, MG. Material e Methods: Quasi-experimental study, of the before and after kind with 35 patients in hemodialysis treatment, with 18 years or more, of both sexes, in dialysis for, at least, two months and who have had, at least, one appointment with the nutritionist in this division. Sociodemographic and clinical data was collected from patient records and adapted reminder questionnaires of food frequency in the last 24 hours were applied for assessment of food intake, as well as multiple-choice questions about the knowledge of the causes of hyperphosphatemia, phosphorus-rich food and use of binders. The intervention was made through the use of an educational leaflet. After the intervention, phosphorus serum levels were verified, taking into consideration hyperphosphatemia values $>5,5 \mathrm{mg} / \mathrm{dL}$. Results: In the sample, $57.1 \%(n=20)$ were male, with mean age of $61.8 \pm 14.3$ years and mean time in dialysis of $61.1 \pm 68.9$ months. The prevalence of hyperphosphatemia before the intervention had been of $60 \%(n=21)$ and at the end of the intervention there was a reduction to $25.7 \%(n=9)$. There was difference in the reduction of phosphorus serum levels when compared to the medians before and after the intervention $[(5.9 \pm 1.3 \mathrm{mg} / \mathrm{dL} ; 4.9 \pm 1.7 \mathrm{mg} / \mathrm{dL} ; \mathrm{p}<0,001)]$. Conclusion: The results showed that the educational intervention possibly contributed to a better understanding and treatment adherence, having a complementary role in the management of hyperphosphatemia in dialysis patients.

Key-words: Food and Nutritional Education; Renal Dialysis; Hyperphosphatemia; Renal Insufficiency, Chronic.
Submetido: $29 / 04 / 2019$ Aceito: $18 / 10 / 2019$ 


\section{INTRODUÇÃO}

A doença renal crônica (DRC) é caracterizada como uma síndrome clínica decorrente de anormalidades da estrutura ou função renal, gerando implicações para a saúde. São várias as causas da DRC, sendo as mais comuns a hipertensão arterial grave, o diabetes mellitus e a glomerulonefrite crônica. ${ }^{1,2}$

A hemodiálise (HD) é a terapia substitutiva para portadores de DRC em estágio final mais empregada na atualidade, ${ }^{3}$ sendo realizada através de um processo extracorpóreo de filtragem e depuração do sangue. Tem como função a eliminação de substâncias tóxicas ao organismo que se acumulam no indivíduo com DRC. ${ }^{2}$ Dentre essas substâncias, temos o fósforo, levando ao quadro de hiperfosfatemia, com alta prevalência entre os pacientes que realizam HD. ${ }^{4}$

Em pacientes portadores de DRC em terapia substitutiva, a hiperfosfatemia pode ocorrer por ingestão de alimentos ricos em fósforo e diminuição da depuração de fósforo através dos processos dialíticos. Também contribui para o surgimento de hiperparatireoidismo secundário (HPS) e de outros distúrbios ósseos, associado com o aumento da morbimortalidade nesta população. 5,6

Como o tratamento dialítico é relativamente ineficiente para a remoção do excesso do fósforo sanguíneo, o controle da ingestão alimentar de fósforo, bem como a utilização de quelantes de fósforo juntamente com as refeições, tem sido uma opção para reduzir a absorção intestinal desse elemento. ${ }^{7-9}$ Por isso, associar o aconselhamento dietético individual com trabalhos de intervenção alimentar e nutricional, é importante para contribuir no melhor entendimento sobre o controle da hiperfosfatemia, evitando possíveis complicações oriundas da DRC. ${ }^{10,11}$

Estudos mostram que a prática de intervenções educacionais é eficaz para o controle da hiperfosfatemia, assim como a sensibilização da equipe interdisciplinar. A promoção de educação continuada e o acompanhamento nutricional adequado nessa população, contribuem para um melhor sucesso na adesão ao tratamento dialítico. 4,5,12

Sendo assim, o objetivo do presente estudo foi descrever, em indivíduos em hemodiálise, o conhecimento do tratamento da hiperfosfatemia, bem como avaliar o possível impacto das orientações nutricionais nos níveis de fósforo após intervenção nutricional em participantes do programa de HD no Hospital Universitário na cidade de Juiz de Fora, MG.

\section{MATERIAL E MÉTODOS}

Trata-se de um estudo quase-experimental, do tipo antes e depois realizado com pacientes que participavam do programa de HD no Hospital
Universitário da Universidade Federal de Juiz de Fora (HU-UFJF/EBSERH), no setor de nefrologia, no turno da manhã. A pesquisa foi aprovada pelo Comitê de Ética em Pesquisa com Seres Humanos do Hospital Universitário da Universidade Federal de Juiz de Fora, sob o número do protocolo 2.262.239. Todos os participantes assinaram o Termo de Consentimento Livre e Esclarecido e a coleta dos dados foi realizada no período de agosto a dezembro de 2016 .

O estudo incluiu uma amostra de conveniência, sendo coletados dados de 35 pacientes em HD (realizada três vezes por semana, tendo duração de quatro horas cada sessão). Os critérios de inclusão no estudo foram pacientes com idade maior ou igual a 18 anos, de ambos os sexos, residentes nos municípios de abrangência do programa de HD, que haviam iniciado o tratamento dialítico há, pelo menos, dois meses e que haviam passado por, pelo menos, uma consulta com a nutricionista do setor para orientações e plano alimentar individualizado. O critério de exclusão do estudo foi pacientes que já haviam realizado cirurgia prévia para remoção da glândula paratireoide (paratireoidectomia).

Foram coletados do prontuário dados sociodemográficos e clínicos dos pacientes para caracterização da população como idade, sexo, raça, escolaridade, estado civil, enfermidades associadas, etiologia da DRC, tempo de diálise (meses) e medicamentos em uso (tipo e frequência), assim como dados de avaliação nutricional, incluindo informações antropométricas como peso seco $(\mathrm{Kg})$ e estatura $(\mathrm{m})$ aferidos em balança e fita do setor para posterior cálculo de índice de massa corporal (IMC), sendo utilizado os pontos de corte da Organização Mundial da Saúde, ${ }^{13}$ onde são classificados como baixo peso com IMC $<18,5$ $\mathrm{Kg} / \mathrm{m}^{2}$, eutrofia IMC entre 18,5 a $24,9 \mathrm{Kg} / \mathrm{m}^{2}$, sobrepeso IMC entre 25 a $29,9 \mathrm{Kg} / \mathrm{m}^{2}$ e obesidade IMC $\geq 30 \mathrm{Kg} / \mathrm{m}^{2}$.

Para a avaliação do consumo alimentar, foi utilizado o recordatório alimentar de $24 \mathrm{~h}$ realizado em dois momentos distintos, um dia de HD e outro dia comum, ambos realizados antes da intervenção, com intervalo de uma semana entre as aplicações. A análise do consumo foi realizada através de tabelas de composição de alimentos - Taco, IBGE e Tucunduva. ${ }^{14-16}$ Além disso, foi aplicado questionário de frequência alimentar adaptado, elaborado a partir do questionário validado por Sichieri \& Everhart contendo 17 itens com alimentos fonte de fósforo de interesse do estudo, uma vez que não existe questionário específico validado para essa população. ${ }^{17}$

Foi também aplicado um questionário que continha questões fechadas sobre o conhecimento das causas da hiperfosfatemia, alimentos ricos em fósforo e uso de quelantes. Todas as questões foram lidas pelo pesquisador para todos os pacientes durante a sessão de diálise, independentemente do grau de escolaridade e todos os questionários foram aplicados antes da realização da intervenção. 
A intervenção foi realizada nos meses de setembro a novembro de 2016, após a aplicação dos questionários, onde foi utilizada uma cartilha educativa com informações simples e de fácil compreensão, contendo imagens ilustrativas e informações sobre as causas da hiperfosfatemia, principais complicações em decorrência dos níveis séricos de fósforo elevados, bem como alimentos ricos em fósforo e orientações acerca do uso correto dos quelantes de fósforo.

As abordagens foram realizadas individualmente, durante a sessão de diálise, com duração média de 40 minutos. Para avaliação da intervenção, foram coletados através dos prontuários, dados de exames bioquímicos, realizados de acordo com a rotina do serviço para controle do fósforo sérico (considerando adequado valores entre $3,5-5,5 \mathrm{mg} / \mathrm{dL}$ ), referentes aos meses de agosto e dezembro de 2016 (antes e após a intervenção). O nível sérico de paratormônio (PTH) também foi coletado mediante prontuário (considerando como valor de referência $<300 \mathrm{mg} / \mathrm{dL}$ ). Entretanto, só foi possível a coleta referente ao mês de outubro (durante a intervenção), uma vez que a dosagem bioquímica desse hormônio é realizada semestralmente, de acordo com a portaria vigente. Ademais, foram verificados valores de Kt/V para avaliação da eficiência da diálise, considerando adequados valores $\geq 1,2 .{ }^{18}$

As análises estatísticas foram realizadas através do software Statistical Package for the Social Sciences (SPSS) versão 23.0. Para verificar a normalidade, utilizou-se o teste de Shapiro Wilk, sendo as variáveis quantitativas descritas através de média e desvio padrão e as variáveis categóricas descritas através de frequências absolutas e relativas. A associação entre as variáveis categóricas foi avaliada pelo Teste McNemar. Para a análise das variáveis quantitativas, foi aplicado o Teste t-Student pareado, para comparação antes e após a intervenção. O nível de significância estatística considerado no estudo foi de $5 \%(p \leq 0,05)$.

\section{RESULTADOS}

A amostra foi composta por 35 pacientes, sendo $57,1 \%(n=20)$ do sexo masculino, com média de tempo de realização de diálise de 61,1 \pm 68,9 meses e Kt/V de $1,55 \pm 0,4$. As características sociodemográficas e clínicas estão descritas na tabela 1 .

Quanto ao estado nutricional, observou-se que $68,6 \%(n=24)$ apresentavam-se em eutrofia, $17,1 \%$ $(n=6)$ com sobrepeso, 8,6\% $(n=3)$ com obesidade e $5,7 \%(n=2)$ com baixo peso, sendo a média de IMC para as mulheres de $23,06 \pm 4,5 \mathrm{Kg} / \mathrm{m}^{2}$ e para os homens de $23,6 \pm 3,4 \mathrm{~kg} / \mathrm{m}^{2}$, ambos dentro da classificação de eutrofia, de acordo com os valores de pontos de corte da OMS. ${ }^{13}$

Todos os pacientes faziam uso de três ou mais medicamentos diferentes por dia, sendo $94,3 \%$ $(n=33)$ da classe dos anti-hipertensivos, 94,3\%
Tabela 1: Características sociodemográficas e clínicas de pacientes renais em tratamento hemodialítico do Hospital Universitário de Juiz de Fora, MG, 2016.

\begin{tabular}{|c|c|}
\hline Variável & $\begin{array}{c}\text { Média } \pm \\
\text { Desvio } \\
\text { padrão \% } \\
(\mathbf{n})\end{array}$ \\
\hline Idade (anos) & $61,8 \pm 14,3$ \\
\hline \multicolumn{2}{|l|}{ Sexo } \\
\hline Masculino & $57,1(20)$ \\
\hline Feminino & $42,9(15)$ \\
\hline \multicolumn{2}{|l|}{ Peso seco $(\mathrm{Kg})$} \\
\hline Masculino & $67,3 \pm 11,3$ \\
\hline Feminino & $54,3 \pm 12,1$ \\
\hline \multicolumn{2}{|l|}{ Estatura (m) } \\
\hline Masculino & $1,68 \pm 0,09$ \\
\hline Feminino & $1,53 \pm 0,06$ \\
\hline \multicolumn{2}{|l|}{ Escolaridade } \\
\hline Analfabeto (a) & $20,0(7)$ \\
\hline Ensino fundamental completo & $45,7(16)$ \\
\hline Ensino médio completo & $20,0(7)$ \\
\hline \multicolumn{2}{|l|}{ Etiologia da Doença Renal Crônica } \\
\hline Nefropatia hipertensiva & $31,4(11)$ \\
\hline Indeterminada & $22,9(8)$ \\
\hline Nefropatia diabética & $17,1(6)$ \\
\hline Glomerulonefrite crônica & $17,1(6)$ \\
\hline Outras causas & $11,5(4)$ \\
\hline \multicolumn{2}{|l|}{ Raça/cor autodeclarada } \\
\hline Branca & $34,3(12)$ \\
\hline Negro & $34,3(12)$ \\
\hline Parda & $31,4(11)$ \\
\hline \multicolumn{2}{|l|}{ Estado civil } \\
\hline Solteiro (a) & $17,1(6)$ \\
\hline Casado (a) & $40,0(14)$ \\
\hline Divorciado/Separado (a) & $17,1(6)$ \\
\hline Viúvo (a) & $25,8(9)$ \\
\hline \multicolumn{2}{|l|}{ Enfermidades associadas } \\
\hline Hipertensão arterial & $94,2(33)$ \\
\hline Cardiopatias & $48,5(17)$ \\
\hline Diabetes mellitus & $34,2(12)$ \\
\hline Hiperparatireoidismo secundário & $28,5(10)$ \\
\hline \multicolumn{2}{|l|}{ Uso de quelantes de fósforo } \\
\hline Sim & $94,3(33)$ \\
\hline Não & $5,7(2)$ \\
\hline
\end{tabular}

$(n=33)$ dos quelantes de fósforo e 34,2\% $(n=12)$ dos hipoglicemiantes. Quanto ao uso do quelante de fósforo, 75,8\% $(n=25)$ utilizavam o Carbonato de Cálcio e apenas $24,2 \%(n=8)$ utilizavam o Sevelamer. Destes que faziam uso do quelante de fósforo, apenas 63,7\% $(n=21)$ usavam conforme a prescrição médica, utilizando o número correto de comprimidos por refeição. Já entre 
aqueles que faziam uso de forma diferente do prescrito $(36,3 \%)$, foi observado que a maioria, $83,3 \% \quad(n=10)$ usava menos comprimidos do que estava prescrito.

A tabela 2 apresenta a média de consumo calórico, proteico, de fósforo e a relação fósforo/proteína pela dieta dos pacientes dialíticos (considerando consumo médio do dia de HD e dia comum, realizados antes da intervenção).

Os alimentos fontes de fósforo mais consumidos mediante resposta ao questionário de frequência alimentar adaptado foram aqueles que também eram fontes de proteína, como carnes em geral e ovos, leite e seus derivados e leguminosas (feijão mais relatado). Cerca de $85,7 \%(n=30)$ da amostra não consumiam alimentos integrais e somente $17,1 \%(n=6)$ relataram o consumo de 1 a 4 vezes na semana que refrigerantes à base de cola.

A tabela 3 mostra a porcentagem de acertos de cada questão sobre o conhecimento das causas da hiperfosfatemia, alimentos ricos em fósforo e uso de quelantes. Com relação à questão que abordava os problemas decorrentes do aumento do fósforo, os pacientes apresentaram bom percentual de acerto, exceto para o problema relacionado ao endurecimento do coração, onde apenas $54,2 \%(n=19)$ da amostra apontou como um problema.

Quando questionados sobre quais alimentos eram ricos em fósforo, os pacientes demostraram bom conhecimento, no entanto, apenas $65,7 \%(n=23)$ responderam que carnes em geral eram alimentos ricos em fósforo e $45,8 \%(n=16)$ da amostra consideraram que as frutas eram ricas em fósforo.

$\mathrm{Na}$ abordagem sobre os quelantes de fósforo, $48,5 \%(n=17)$ não souberam qual medicação utilizada era o quelante e $60 \%(n=21)$ relataram não saber para que serve o mesmo. Quanto ao uso dos quelantes, $87,9 \%(n=29)$ acertaram o momento em que se deve tomar o quelante, junto com as refeições e apenas $33,3 \%(n=11)$ responderam que não fazem uso do mesmo quando não realizam as refeições.

A prevalência de hiperfosfatemia entre os pacientes antes da intervenção era de $60 \%(n=21)$ e ao

Tabela 2: Média de consumo calórico, proteico, de fósforo e relação fósforo/proteína pela dieta de pacientes renais em tratamento hemodialítico do Hospital Universitário de Juiz de Fora, MG, 2016.

\begin{tabular}{cc}
\hline Variável & Média \pm Desvio Padrão \\
\hline Fósforo/dia $(\mathrm{mg})$ & $797,3 \pm 203,5$ \\
\hline Proteína/dia $(\mathrm{g})$ & $63,8 \pm 17,7$ \\
\hline Proteína/Kg/dia $(\mathrm{g})$ & $1,1 \pm 0,4$ \\
\hline $\mathrm{Kcal} / \mathrm{dia}$ & $1544,6 \pm 356,7$ \\
\hline $\mathrm{Kcal} / \mathrm{Kg} / \mathrm{dia}$ & $26,0 \pm 8,0$ \\
\hline $\begin{array}{c}\text { Fósforo/Proteína/dia } \\
(\mathrm{mg} / \mathrm{g})\end{array}$ & $12,8 \pm 2,5$ \\
\hline
\end{tabular}

final da intervenção houve redução para $25,7 \%(n=9)$, apresentando diferença $(p=0,004)$.

A média dos níveis séricos de PTH foi de 491,3 $\pm 618,9 \mathrm{mg} / \mathrm{dL}$, estando $42,8 \%(n=15)$ da amostra com níveis acima dos valores de referência. Observou-se redução dos níveis de fósforo sérico, quando comparado o período inicial (P0) e final (P1), com diferença $(p<0,001)$. A tabela 4 descreve os níveis séricos antes e após a intervenção dos parâmetros bioquímicos.

\section{DISCUSSÃO}

No presente estudo, foi verificado que mais da metade dos pacientes em HD apresentavam quadro de hiperfosfatemia. Apesar de receberem orientações nutricionais mensais e plano alimentar individualizado, a prevalência de hiperfosfatemia se mostrou bastante significativa.

Na amostra, 34,2\% encontravam-se em diálise há mais de cinco anos, achado semelhante ao estudo de Pascoal et $\mathrm{al}^{12}(40,5 \%)$ e Vaz et $\mathrm{al}^{19}(37,2 \%)$. Estudos mostram que, independente do tempo de realização de HD, a adesão e o comprometimento no controle da hiperfosfatemia ainda é um desafio. ${ }^{12,20}$

No presente estudo, o carbonato de cálcio $(75,8 \%)$ foi o quelante de fósforo mais utilizado, achado esse, diferente do exposto pelo "Inquérito Brasileiro de Diálise Crônica 2016", onde apenas 26\% dos pacientes em HD no país utilizam carbonato de cálcio/acetato de cálcio. ${ }^{3}$ Observou-se que boa parte não possuía conhecimento sobre a função do medicamento. Apesar de aproximadamente $88 \%$ dos pacientes relatarem que ingerem a medicação logo após as refeições, muitos utilizavam menos comprimidos do que o prescrito pelo médico, o que pode contribuir para uma maior absorção de fósforo, e, consequentemente, aumento dos níveis séricos deste mineral. Como a diminuição dos níveis de fósforo sérico não ocorre de forma rápida, isso favorece a desmotivação e descontinuação do tratamento. ${ }^{4}$

Com relação à questão que abordava os problemas ocasionados pela hiperfosfatemia, apenas $54,2 \%$ dos pacientes relataram saber que níveis altos no sangue levariam ao "endurecimento do coração". Valores parecidos também foram encontrados no estudo de Nerbass et $\mathrm{al}^{9}$, onde apenas $52 \%$ acertaram essa questão. Já no estudo de Brito et $\mathrm{al}^{4}$, esse percentual foi maior, cerca de $68 \%$ da amostra. Quando questionados sobre os alimentos ricos em fósforo, os pacientes demonstraram bom conhecimento. Entretanto, mais da metade dos pacientes responderam que os alimentos ricos em potássio, como as frutas em geral, eram alimentos ricos em fósforo, mostrando a dificuldade em assimilar e diferenciar os alimentos ricos em fósforo e potássio.

Quando analisados o consumo alimentar, observou-se que a ingestão calórica, proteica e de fósforo ficaram abaixo dos valores recomendados para 
Tabela 3: Análise do conhecimento sobre hiperfosfatemia e uso de quelantes de fósforo de pacientes renais em tratamento hemodialítico do Hospital Universitário de Juiz de Fora, MG, 2016.

$\%$ Acertos (n)

Que problemas o fósforo alto pode causar?

Coceiras em todo o corpo

$85,7(30)$

Endurecimento do coração

$54,2(19)$

Dores e fraqueza nos ossos

$74,2(26)$

Aumento do risco de morte

$77,1(27)$

Média de acertos

72,8

Quais dos alimentos são ricos em fósforo?

Leite e seus derivados (iogurtes e queijos)

$71,4(25)$

Frutas em geral (banana, laranja e mamão)

$45,7(16)$

Embutidos (linguiça, presunto e mortadela)

$82,8(29)$

Carnes (boi, frango e peixes)

$65,7(23)$

Refrigerantes tipo cola e cerveja

$82,8(29)$

Sucos de frutas

$71,4(25)$

Média de acertos

Qual a medicação que você toma é o quelante de fósforo?

Carbonato de Cálcio/ Sevelamer

$45,4(15)$

Outras medicações

$6,1(2)$

Não souber responder

$48,5(16)$

Sabe para que serve o quelante de fósforo?

Sim

$40(13)$

Não

$60(20)$

Em que momento o quelante de fósforo deve ser tomado?

Junto com as refeições

$87,9(29)$

Longe das refeições

$12,1(4)$

Tabela 4: Parâmetros bioquímicos antes e após a intervenção de pacientes renais em tratamento hemodialítico do Hospital Universitário de Juiz de Fora, MG, 2016.

\begin{tabular}{cccc}
\hline Variável & Antes da intervenção & Após a intervenção & p-valor \\
\hline Fósforo $(\mathrm{mg} / \mathrm{dL})$ & $5,9 \pm 1,3$ & $4,9 \pm 1,7$ & $<0,001^{*}$ \\
\hline Cálcio Iônico $(\mathrm{mg} / \mathrm{dL})$ & $9,2 \pm 1,0$ & $9,9 \pm 0,9$ & $<0,001^{*}$ \\
\hline
\end{tabular}

*Teste T pareado $p<0,05$. Nota: Os dados são apresentados como média \pm desvio padrão.

essa população. 2,21,22 Apenas a relação fósforo/proteína ficou dentro das recomendações estabelecidas pelo KDOQI (de 10 a $16 \mathrm{mg} / \mathrm{g}$ de proteína do alimento) como forma de auxílio e controle do fósforo na dieta. ${ }^{23}$ Apesar de não se ter avaliado a ingestão de fósforo proveniente de alimentos industrializados, sabe-se que estes produtos alimentícios também contribuem para a maior ingestão diária de fósforo e aumento dos níveis séricos no organismo.

No estudo de Pinto et $\mathrm{al}^{10}$, onde foi avaliada a ingestão calórica, proteica e de fósforo, verificou-se que a média para consumo calórico foi de $28 \mathrm{Kcal} / \mathrm{Kg} / \mathrm{dia}$, de proteína $1,1 \mathrm{~g} / \mathrm{Kg} /$ dia e de fósforo $958 \mathrm{mg} / \mathrm{dia}$, valores próximos ao encontrado no nosso estudo. Diferente do encontrado por Machado et $\mathrm{al}^{24}$, onde o consumo calórico, proteico e de fósforo foi de $19 \mathrm{kcal} / \mathrm{kg} / \mathrm{dia}, 0,9$ $\mathrm{g} / \mathrm{Kg} /$ dia de proteína e $612,5 \mathrm{mg} /$ dia de fósforo.

Estudo publicado recentemente por Guida et al $^{25}$ mostrou que a substituição de alimentos proteicos com menor relação fósforo/proteína na dieta de pessoas em hemodiálise favoreceu o controle do fósforo sérico, sem alterar o peso corporal.

Foi observado que antes de passarem pela intervenção, 60\% da amostra apresentavam alteração nos níveis séricos de fósforo, diferente do encontrado por Brito et $\mathrm{al}^{4}$ em seu estudo, onde apenas $36 \%$ da amostra apresentavam elevação deste mineral. Após a intervenção, houve redução significativa da 
hiperfosfatemia quando comparados os períodos M0 e M1, onde apenas $25 \%$ permaneceram com o fósforo sérico elevado. Entretanto, a alta prevalência de hiperfosfatemia nesses pacientes pode ser justificada pela presença de doença óssea avançada, não sendo exclusivamente decorrente de uma alimentação inadequada, uma vez que $42,8 \%$ dos pacientes apresentam alterações de PTH.

Danelon et al26 observaram resultados significativos na redução do fósforo sérico após intervenções nutricionais em pacientes em hemodiálise, corroborando para importância da realização dessas atividades no tratamento da hiperfosfatemia.

Nisio et $a^{27}$ também demostraram em seu estudo resultados positivos na redução do fósforo sérico de pacientes em HD quando submetidos a um programa de educação nutricional, também utilizando material didático lúdico e de fácil compreensão. Outros estudos também demonstraram resultados positivos com a utilização de práticas educativas nutricionais como forma de auxílio no tratamento da hiperfosfatemia e qualidade de vida. ${ }^{12,28,29}$ De fato, uma revisão sistemática confirmou que realizar atividades de educação alimentar e nutricional promove a redução significativa do fósforo sérico no grupo de intervenção. ${ }^{30}$ Caso a mudança não ocorra, há o ganho de conhecimento quanto aos aspectos nutricionais uma vez que as mudanças laboratoriais podem demandar mais tempo de monitoramento para serem detectadas. ${ }^{31}$

O presente estudo apresenta limitações como o fato de não ter sido avaliado a ingestão de alimentos industrializados ricos em conservantes e estabilizantes a base de fósforo o que pode influenciar numa maior ingestão de fósforo diária, e consequentemente, nos níveis séricos de fósforo no organismo. Ademais, por se tratar de um estudo quase-experimental, do tipo antes e depois e a não realização dos questionários e recordatório alimentar $24 \mathrm{~h}$, no pós-intervenção, foram outros pontos limitantes do estudo, reduzindo o poder do mesmo. Entretanto, a sua realização não foi possível devido a não compatibilidade de horários e falta de pessoal de apoio. Além disso, os níveis séricos de fosfato sofrem outras influências além da alimentação, como modificações nos parâmetros da diálise, ajuste no uso de quelantes, de análogos da vitamina $D$ e calcimiméticos. Neste estudo, não foi possível avaliar tais variáveis de confusão.

\section{CONCLUSÃO}

A intervenção em educação alimentar e nutricional focada nos níveis de fósforo dos alimentos, no incentivo ao uso correto dos quelantes de fósforo e nas possíveis complicações da hiperfosfatemia, possivelmente contribuem para uma melhor compreensão e adesão ao tratamento, auxiliando na diminuição dos níveis séricos de fósforo. Apesar de ter sido positiva a intervenção educativa, ressalta-se a importância de realizar ações de forma continuada, em conjunto com o acompanhamento nutricional individualizado, o ajuste nas prescrições de diálise, de análogos de vitamina $D$ e calcimiméticos. Assim, os pacientes serão sempre conscientizados da importância do tratamento, visto que os pacientes em HD apresentam diversas restrições, o que por muitas vezes gera confusões e esquecimento.

\section{FINANCIAMENTO}

O estudo não recebeu financiamento.

\section{CONFLITO DE INTERESSES}

Os autores declaram não haver conflito de interesses.

\section{REFERÊNCIAS}

1. National Kidney Foundation. K/DOQI US Commentary on the 2012 KDIGO Clinical Practice Guideline for the Evaluation and Management of CKD. Am J Kidney Dis. 2014; 63(5):713-35. doi: https://doi.org/10.1053/j.ajkd.2014.01.416

2. Cuppari L. Nutrição clínica no adulto: guias de medicina ambulatorial e hospitalar UNIFESP/Escola Paulista de Medicina. 3a ed. São Paulo: Ed. Manole; 2014.

3. Sesso RC, Lopes AA, Thomé FS, Lugon JR, Martins CT. Inquérito brasileiro de diálise crônica 2016. J Bras Nefrol. 2017; 39(3):261-6. doi: 10.5935/0101-2800.20170049

4. Brito ACD, França AKTC, Hortegal EV, Dias RSC, Costa RCO, Lima DP. Conhecimento de hiperfosfatemia e quelante de fósforo em hemodialíticos. BRASPEN J. 2016; 31(4):322-8.

5. Caldeira D, Amaral T, David C, Sampaio C. Educational strategies to reduce serum phosphorus in hyperphosphatemic patients with chronic kidney disease: systematic review with meta-analysis. J Ren Nutr. 2011; 21(4):285-94. doi: 10.1053/j. jrn.2010.11.006

6. Sherman RA. Hyperphosphatemia in dialysis patients: beyond nonadherence to diet and binders. Am J Kidney Dis. 2016; 67(2):182-6. doi: 10.1053/j.ajkd.2015.07.035

7. Elliott JO, Ortman C, Almaani S, Lee $\mathrm{YH}$, Jordan K. Understanding the associations between modifying factors, individual health beliefs, and hemodialysis patients' adherence to a low-phosphorus diet. J Ren Nutr. 2015; 25(2):111-20. doi: 10.1053/j.jrn.2014.08.006

8. Taketani Y, Koiwa F, Yokoyama K. Management of phosphorus 
load in CKD patients. J Clin Exp Nephrol. 2017; 21(S1):27-36. doi: 10.1007/s10157-016-1360-y

9. Nerbass FB, Morais JG, Santos RG, Krüger TS, Koene TT, Filho HAL. Adesão e conhecimento sobre o tratamento da hiperfosfatemia de pacientes hiperfosfatêmicos em hemodiálise. J Bras Nefrol. 2010; 32(2):149-55. doi: http://dx.doi. org/10.1590/S0101-28002010000200003

10. Pinto DE, Ullmann LS, Burmeister MM, Antonello ICF, Pizzato A. Associações entre ingestão energética, proteica e de fósforo em pacientes portadores de doença renal crônica em tratamento hemodialítico. J Bras Nefrol. 2009; 31(4):269-76. doi: http:// dx.doi.org/10.1590/S0101-28002009000400005.

11. D'Alessandro C, Piccoli GB, Cupisti A. The "phosphorus pyramid": a visual tool for dietary phosphate management in dialysis and CKD patients. BMC Nephrol. 2015; 20(16):9. doi: 10.1186/1471-2369-16-9.

12. Pascoal BA, Paula MR, Maniglia FP. Educação nutricional como estratégia no controle da hiperfosfatemia e hipercalemia em pacientes em hemodiálise. BRASPEN J. 2017; 32(3):221-5.

13. World Health Organization (WHO). Obesity: preventing and managing the global epidemic. Geneva: World Health Organization; 1997.

14. Tabela Brasileira de Composição de Alimentos (TACO). NEPAUNICAMP. 4. ed. rev. e ampl. Campinas: NEPA/UNICAMP; 2011.

15. Instituto Brasileiro de Geografia e Estatística (IBGE). Pesquisa de orçamentos familiares: tabela de composição nutricional dos alimentos consumidos no Brasil. Rio de Janeiro: IBGE; 2011.

16. Philippi ST. Tabela de Composição de Alimentos: Suporte para decisão nutricional. 2a ed. São Paulo: Coronário; 2002.

17. Schieri R, Everhart JE. Validity of a Brazilian food frequency questionnaire against dietary recalls and estimated energy intake. Nutrition. 1998; 18(10):1649-59. doi: https://doi. org/10.1016/S0271-5317(98)00151-1

18. National Kidney Foundation. K/DOQI Clinical Practice Guideline for Hemodialysis Adequacy: 2015 update. Am J Kidney Dis. 2015; 66(5):884-930. doi: https://doi.org/10.1053/j. ajkd.2015.07.015

19. Vaz IMF, Freitas ATVS, Peixoto MRG, Ferraz SF, Campos MIVA. A ingestão energética de pacientes em hemodiálise é subrelatada? J Bras Nefrol 2015;37(3):359-66. doi: http:// dx.doi.org/10.5935/0101-2800.20150056.

20. Hoover $\mathrm{H}$. Compliance in hemodialysis patients: a review of the literature. J Am Diet Assoc. 1989; 89(7):957-9.
21. National Kidney Foundation. KDOQI US Commentary on the 2017 KDIGO Clinical Practice Guideline Update for the Diagnosis, Evaluation, Prevention, and Treatment of Chronic Kidney Disease-Mineral and Bone Disorder (CKD-MBD). Am J Kidney Dis. 2017; 70(6):737-51. doi: 10.1053/j.ajkd.2017.07.019.

22. National Kidney Foundation. K/DOQI Clinical practice guidelines for nutrition in chronic renal failure. Am J Kidney Dis. 2001; 37(1 Suppl 2):S66-70.

23. National Kidney Foundation. K/DOQI Clinical practice guidelines for bone metabolism and disease in chronic kidney disease. Am J Kidney Dis. 2003; 42(4 Suppl 3):S1-201.

24. Machado AD, Bazanelli AP, Simony RF. Avaliação do consumo alimentar de pacientes com doença renal crônica em hemodiálise. Rev Ciênc. Saúde. 2014; 7(2):76-84. doi: http:// dx.doi.org/10.15448/1983-652X.2014.2.17758.

25. Guida B, Parolisi S, Coco M, Ruoppo T, Veccia R, di Maro $M$ et al. The impact of a nutritional intervention based on egg white for phosphorus control in hemodialyis patients. Nutr Metab Cardiovasc Dis. 2019; 29(1):45-50. doi: 10.1016/j. numecd.2018.09.010

26. Danelon B, Andrade MM, Alvarenga L, Nascimento $R$, Mendes LL, Aguiar A. Efeitos a curto e longo prazos de ações de educação alimentar e nutricional no perfil nutricional de pacientes em hemodiálise. Nutr clín diet hosp. 2018; 38(4):1316. doi: 10.12873/384danelon.

27. Nisio JM, Bazanelli AP, Kamimura MA, Lopes MGG, Ribeiro FSM, Vasselai $P$ et al. Impacto de um programa de educação nutricional no controle da hiperfosfatemia de pacientes em hemodiálise. J Bras Nefrol. 2007; 29(3):152-57.

28. Baraz S, Zarea K, Dashtbozorgi B. Comparing the effect of two educational programs on the quality of life of hemodialysis patients in Iran. Iran Red Crescent Med J. 2014. 16(8):e19368. doi: $10.5812 / \mathrm{ircmj} .19368$.

29. Ebrahimi H, Sadeghi M, Amanpour F, Dadgari A. Influence of nutritional education on hemodialysis patients' knowledge and quality of life. Saudi J Kidney Dis Transpl. 2016; 27(2):250-5. doi: $10.4103 / 1319-2442.178253$.

30. Karavetian M, de Vries N, Rizk R, Elzein H. Dietary educational interventions for management of hyperphosphatemia in hemodialysis patients: a systematic review and meta-analysis. Nutr Rev. 2014 Jul; 72(7):471-82. doi: 10.1111/nure.12115. Epub 2014 Jun 11.

31. Casas J, Rodrigues CIS, D'Avila R. Educação nutricional para pacientes renais crônicos em programa de hemodiálise. Nutrire. 2015; 40(1):36-44. 